\title{
Magneto-Optical Beam Splitter for Atoms
}

\author{
T. Pfau, Ch. Kurtsiefer, C. S. Adams, M. Sigel, and J. Mlynek \\ Fakultät für Physik, Universität Konstanz, D-78434 Konstanz, Germany \\ (Received 21 July 1993)
}

\begin{abstract}
We report an experimental demonstration of diffraction of $\mathrm{He}^{*}$ atoms from a magneto-optical grating. The grating was produced by the interaction of three-level atoms with a light field of spatially varying polarization and a magnetic field. For a light shift matched to the Zeeman shift, a two-peaked diffraction pattern was observed, corresponding to a momentum splitting of $42 \hbar k$. The effect of changing the polarizations of the light field was investigated. The diffraction from the magneto-optical grating is compared directly with the diffraction of two-level atoms from a standing light wave and it is shown that the magneto-optical interaction leads to more efficient coupling into high order momentum states.
\end{abstract}

PACS numbers: $32.80 .-\mathrm{t}, 41.85 .-\mathrm{p}, 42.50 . \mathrm{Vk}$

The diffraction of atomic matter waves from a periodic structure was first demonstrated by Stern in 1929 [1]. Although the primary interest at that time was the demonstration of the wave nature of matter, the experiment was the first demonstration of an atomic beam splitter using a diffraction technique. Recently coherent beam splitters for atoms found new interest as key elements in atom optical systems like atom interferometers, or as an input/output coupler in atomic cavities. Present atom interferometers are based on diffraction from free standing transmission structures [2,3], an optical four zone Ramsey technique [4], on a sequence of Raman pulses [5], or the magnetic Stern-Gerlach effect [6].

The optical techniques make use of the momentum transferred to an atom by photon recoil, in order to coherently split and recombine the atomic wave function. The transverse splitting angle of such beam splitters is limited to one [4] or two [5] photon momenta divided by the longitudinal atomic momentum. In Mach-Zehnder type interferometers a large splitting angle is required for a clear spatial beam separation and in some applications for an increased sensitivity. Future developments will aim at new methods to transfer considerably larger momentum during the splitting process. Larger splitting angles have been achieved, e.g., by using the optical Stern-Gerlach effect [7] or a sequence of Raman pulses [8]. Another technique to produce significant momentum transfer is the diffraction of atoms from a standing wave light field [9]. In this paper we report on the experimental demonstration of the coherent diffraction of a beam of metastable helium atoms from a magneto-optical grating. To our knowledge we achieved the largest splitting in momentum space observed so far.

If spontaneous emission is avoided and if the atom stays adiabatically in one eigenstate, the light-atom interaction leads to a transversely varying phase shift on the atomic wave function. It can therefore be regarded as diffraction of an atomic wave function from a phase grating. If the light-induced kinetic energy change of the atom is small compared to the coupling energies to the light field (Raman-Nath approximation), the phase modulation is given by the line integral of the optical po- tential along the beam direction. The diffraction process for an incoming atomic plane wave is schematically displayed in Fig. 1(a). The interaction of a three-level atom with the magneto-optical configuration is analogous to diffraction from a phase grating with an approximately triangularly shaped modulation. For high modulation amplitudes the magneto-optical phase grating produces a clearly two-peaked splitting in momentum space, i.e., an efficient diffraction into high order momentum states. This is in contrast to the interaction of a two-level atom with a standing wave light field, where the spatial modulation of the phase grating is approximately sinusoidal.

We briefly describe the theory of the coherent magneto-optical diffraction process using an atom with a $V$ level scheme. The formalism is described in more detail

(a)

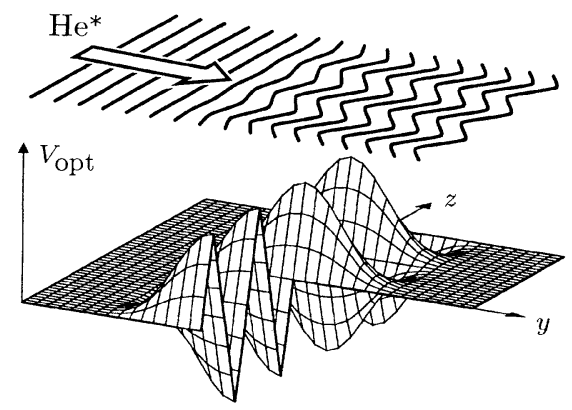

(b)

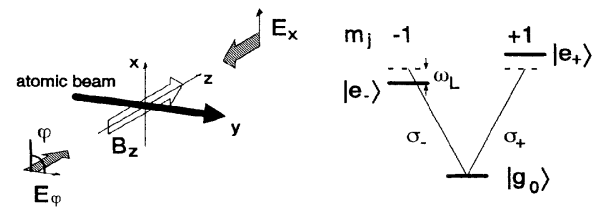

FIG. 1. (a) Diffraction process in a Gaussian laser beam for an incoming plane atomic wave. The outgoing beam contains two almost plane waves. (b) Schematic configuration of the counterpropagating laser beams and the magnetic field interacting with the three-level atoms in the atomic beam. 
in earlier work $[10,11]$. By "coherent" we refer to the case where the probability of spontaneous emission is very low.

We consider a $J=1 \rightarrow J^{\prime}=1$ transition and choose the quantization axis parallel to the magnetic field direction, i.e., along the $z$ axis. The population can be optically pumped into the $\left|g_{0}\right\rangle$ ground state. For light propagating along the quantization axis $\Delta m_{J}=0$ transitions are absent and the level scheme reduces to a $V$ system [Fig. 1(b)]. The magnetic interaction energy is $H_{B}=-\boldsymbol{\mu} \cdot \mathbf{B}$, where $\mu=-g_{J} \mu_{B} J$. The magnetic field is assumed to be homogeneous. The atom-laser interaction energy in the electric dipole approximation is given by $H_{A-L}$ $=-\mathbf{d} \cdot \operatorname{Re}\left\{\mathbf{E}(\mathbf{r}) e^{-i \omega t}\right\}$, where $\mathbf{d}$ is the electric dipole operator and $\mathbf{E}(\mathbf{r})$ the laser field:

$$
\mathrm{E}(\mathrm{r})=E_{0}(x, y)\left[\left(\hat{\boldsymbol{\epsilon}}_{x} \cos \phi+\hat{\boldsymbol{\epsilon}}_{y} \sin \phi\right) e^{i k z}+\hat{\boldsymbol{\epsilon}}_{x} e^{-i k z}\right] .
$$

This field is produced by two counterpropagating linearly polarized laser beams with $k$ vector along the $z$ axis, with one polarization in the $x$ direction $\left(\hat{\epsilon}_{x}\right)$ and one tilted by an angle $\phi$ in the $x y$ plane. The spatial dependence of the field amplitude $E_{0}(x, y)$ on $y$ can, in the moving frame of the atom, be written as an explicit time dependence $t=y / v_{y}$, where $v_{y}$ is the group velocity of the atoms, which propagate in the $y$ direction (Fig. 1). For simplicity we assume that the field is uniform along $x$.

The three eigenvalues of the interaction Hamiltonian in the rotating wave approximation and for zero laser detuning $\delta$ (i.e., the laser frequency $\omega$ is equal to the unperturbed transition frequency) are given by

$$
\mathscr{E}_{j}=2 \hbar\left(\frac{B}{3}\right)^{1 / 2} \cos \left\{\frac{1}{3} \arccos \left[\frac{c}{4(B / 3)^{3 / 2}}\right]+\frac{2 \pi}{3} j\right\},
$$

where $2 B=2 \omega_{L}^{2}+\omega_{R}^{2}(1+\cos \phi \cos 2 k z), \quad C=\omega_{L} \omega_{R}^{2} \sin \phi$ $\times \sin 2 k_{z}$, and $j=1,2,3$. Here the one beam Rabi frequency is defined as $\omega_{R}(t)=\left\langle e_{ \pm}\left|\mathbf{d} \cdot \epsilon_{ \pm}\right| g_{0}\right\rangle E_{0}\left(v_{y} t\right) / \hbar$ and $\hbar \omega_{L}=g_{J^{\prime}} \mu_{B} B$ is the Zeeman splitting of the excited state levels $\left|e_{ \pm}\right\rangle$. $\hat{\boldsymbol{\epsilon}}_{ \pm}$are unit vectors for $\sigma_{ \pm}$polarized light.

For a particular ratio between the Rabi and the Larmor frequency (given by $\omega_{R}=2 \omega_{L} / \sin \phi$ ), the dressed levels show a degeneracy at positions of $\sigma_{ \pm}$polarized light. This leads to an approximately triangular shape for the magneto-optical potential of the central eigenstate $|j=2\rangle$ (see thicker line in Fig. 2). The energy level structures for $\phi=90^{\circ}$ and $\omega_{R}=2 \omega_{L}$ and for $\phi=45^{\circ}$ and $\omega_{R}$ $=2 \sqrt{2} \omega_{L}$ are shown in Figs. $2(\mathrm{a})$ and $2(\mathrm{~b})$, respectively. By changing the sign of $\phi$ or the magnetic field $B$ the symmetry is reversed.

In the experiment the interaction region is defined by the Gaussian laser profile. For $\omega_{R} \gg 1 / t_{\text {int }}$, where $t_{\text {int }}$ is the interaction time, the switch-on time of the interaction is slow compared to the characteristic time scale of the magneto-optical process $\left(\omega_{L}^{-1}\right.$ or $\left.\omega_{R}^{-1}\right)$ and the evolution

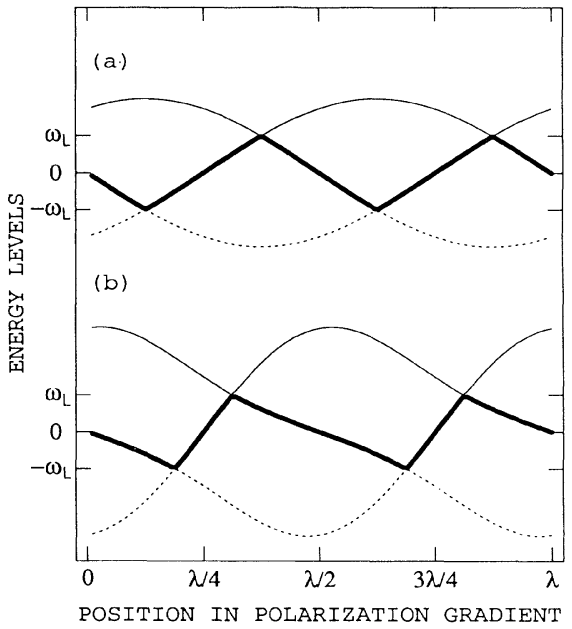

FIG. 2. Spatial dependence of the atomic eigenenergy levels of a $V$ system for (a) $\varphi=90^{\circ}$ and $\omega_{R}=2 \omega_{L}$ and (b) $\varphi=45^{\circ}$ and $\omega_{R}=2 \sqrt{2} \omega_{L}$.

of the atomic internal state is predominantly adiabatic. Hence, an atom entering the interaction region in the ground state $\left|g_{0}\right\rangle$ evolves into the dressed eigenstate $|j=2\rangle$, interacts with the triangular potential, and evolves back to the ground state.

A second consequence of the Gaussian laser profile and the homogeneous magnetic field is that the magnetooptical matching condition is only met at certain positions in the Gaussian profile. The integrated phase shift shows the best triangular modulation for $\omega_{R_{\max }} \simeq 2.3 \omega_{L}$. A schematic representation of the diffraction of an incoming plane wave from the optical potential is shown in Fig. 1 (a).

In the Raman-Nath approximation an incoming atomic plane wave in $\left|g_{0}\right\rangle$ accumulates a spatially varying phase shift $\theta(z)=\int \mathscr{E}_{2}(t) d t / \hbar$. The momentum distribution is then given by $\rho(p) \propto\left|\mathcal{F}\left[e^{i \theta(z)}\right]\right|^{2}$, where $\mathcal{F}$ denotes the Fourier transform with respect to $z$. In the experiment the far-field real space distribution rather than the momentum distribution was measured. Thus the finite velocity distribution and the finite collimation must be included.

The experiment was performed on an atomic beam machine designed for use with metastable rare gas atoms [12]. A supersonic expansion nozzle at room temperature produced a beam of $\mathrm{He}$ atoms, which then were excited into the two metastable states $2^{1} S_{0}$ and $2{ }^{3} S_{1}$ by electron impact at an energy of about $50 \mathrm{eV}$. A quench lamp was used to pump more than $99 \%$ of the metastable singlet atoms to the electronic ground state. The metastable atoms were detected by a channeltron detector. The metastable triplet beam had an average velocity $v_{y}$ $\simeq 2000 \mathrm{~m} / \mathrm{s}$ and a standard deviation of $\Delta v \simeq 280 \mathrm{~m} / \mathrm{s}$.

The incoming beam was collimated by two slits of width 20 and $10 \mu \mathrm{m}$ placed 85 and $1.5 \mathrm{~cm}$ upstream from the interaction region, respectively (see Fig. 3). The 


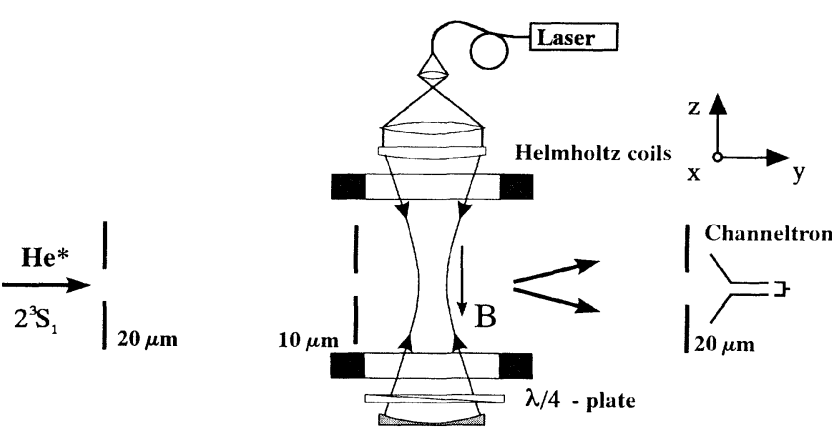

FIG. 3. Experimental configuration for the diffraction of atoms from a magneto-optical grating.

transverse beam profile was measured, $85 \mathrm{~cm}$ downstream from the interaction region, using a $20 \mu \mathrm{m}$ scanning slit in front of the detector. The spatial resolution in the detector plane was equivalent to $0.7 \hbar k$. The dark count rate was below $1 / \mathrm{min}$. All slits were $3 \mathrm{~mm}$ high and were aligned parallel to one another using an optical diffraction technique. A transverse magnetic field was produced by a pair of Helmholtz coils.

The triplet $\mathrm{He}^{*}$ atoms interacted with a light field at a wavelength of $1.083 \mu \mathrm{m}$ resonant with the $2{ }^{3} S_{1}$ to $2{ }^{3} \mathrm{P}$ transition. The light was obtained from a Ti-sapphire ring laser. For the diffraction from the magneto-optical grating the laser was stabilized on the $2{ }^{3} S_{1}$ to $2{ }^{3} P_{1}$ transition using saturation spectroscopy in a dc helium discharge. The atoms were optically pumped into the $\left|g_{0}\right\rangle$ state by a light beam with $\pi$ polarization incident just before the interaction region (not shown in Fig. 3). An electro-optical modulator was used to compensate for the Zeeman shift of the $\pi$ transitions.

The laser beam was brought to the experiment using a single mode optical fiber. The light was passed through a polarizer and expanded by a telescope to produce a parallel Gaussian beam with a diameter of $4 \mathrm{~mm}$. It was then focused in the $y$ direction by a cylindrical lens to a waist of $w_{y}=21 \mu \mathrm{m}$ in the plane of the atomic beam. A cylindrical mirror was used to retroreflect the light. The polarization was controlled by double pass through a $\lambda / 4$ plate in front of the mirror (see Fig. 3). The overlap of the two counterpropagating beams was optimized by monitoring the feedback into the fiber. For the mean velocity in the beam of $v_{y} \simeq 2000 \mathrm{~m} / \mathrm{s}$, the light-atom interaction time was $2 w_{0} / v_{y}=21 \mathrm{~ns}$, i.e., significantly shorter than the $2^{3} P_{1,2}$ state natural lifetime of $99.5 \mathrm{~ns}$. The probability of spontaneous emission therefore was less than $10 \%$. The laser power at the atomic beam was 40 $\mathrm{mW}$ corresponding to a one beam Rabi frequency $\omega_{R} / 2 \pi=340 \mathrm{MHz}$.

In Fig. 4(a) the diffraction pattern in the detector plane for a fixed angle $\varphi=90^{\circ}$ is displayed. It was obtained using a magnetic field of $B=6.7 \mathrm{mT}$, corresponding to a Larmor frequency of $141 \mathrm{MHz}$. Therefore the optimum ratio between $\omega_{R}$ and $\omega_{L}$ was confirmed. For

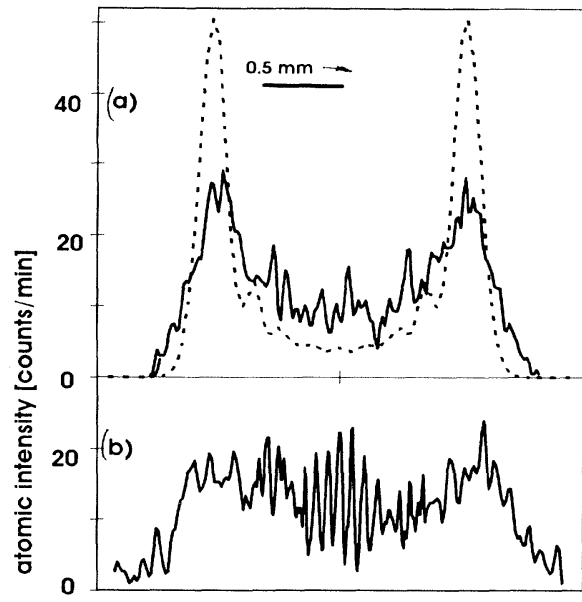

detector position

FIG. 4. (a) Atomic intensity in the detector plane for the magneto-optical beam splitter with $\varphi=90^{\circ}, B=6.7 \mathrm{mT}$, and $\delta / 2 \pi=0 \mathrm{MHz}$. (b) Atomic intensity in the detector plane for standing wave diffraction, $\delta / 2 \pi=-120 \mathrm{MHz}$ and $B=0 \mathrm{mT}$.

these parameters the calculated number of nonadiabatic transitions was less than 0.07 , confirming the validity of the adiabatic approximation [11].

As optical pumping leads to a momentum diffusion of $\sqrt{2} \hbar k$, the individual diffraction orders are not resolved. The distribution is clearly two peaked with a spatial splitting of $1.5 \mathrm{~mm}$, corresponding to a momentum splitting of $42 \hbar k$. Because of the finite velocity distribution, the peaks of the real space distribution are significantly broader than the expected distribution for a monochromatic atomic beam [dashed line in Fig. 4(a)]. Effects of minor importance like imperfect optical pumping, residual nonadiabatic transitions, and spontaneous emission also affect the shape of the momentum distribution.

For comparison we show a standing wave diffraction pattern in Fig. 4(b) $\left(\varphi=0^{\circ}\right)$. For this result the laser was stabilized on the ${ }^{3} S_{1}$ to ${ }^{3} P_{2}$ transition with a detuning of $\delta / 2 \pi=-120 \mathrm{MHz}$. The detuning guarantees adiabatic evolution of the internal atomic state. The magnetic field was turned off and no optical pumping was performed. The $J=1$ to $J=2$ transition behaves like an effective two-level system, because for linearly polarized light, the coupling strengths of the magnetic sublevels to the respective excited state sublevels differ by only $15 \%$. In the center of the distribution a well-resolved $2 \hbar k$ structure was obtained, which disappears for high diffraction orders as a consequence of the finite velocity distribution of the beam.

The effect of rotating the linear polarization of the retroreflected laser beam to angles $\varphi= \pm 45^{\circ}$ is shown in Fig. 5. The ratio of Rabi and Larmor frequency was 340 $\mathrm{MHz} / 92 \mathrm{MHz}=3.7$. This ratio is larger than the matching condition $\omega_{R}=2 \sqrt{2} \omega_{L}$, but the optical potential still 


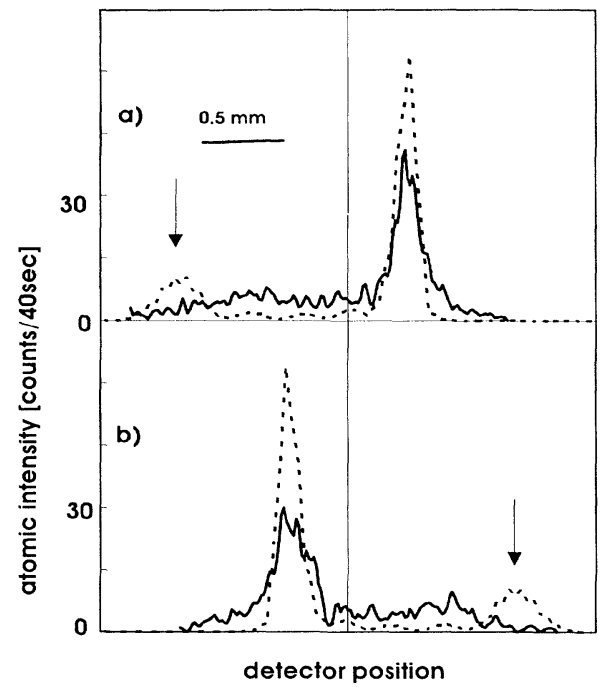

FIG. 5. Atomic intensity in the detector plane for the asymmetric magneto-optical configuration with $B=4.4 \mathrm{mT}, \delta / 2 \pi=0$ $\mathrm{MHz}$ for (a) $\varphi=+45^{\circ}$ and (b) $\varphi=-45^{\circ}$, respectively.

displays an asymmetry [see Fig. 2(b)], leading to an asymmetric diffraction pattern [11]. The dashed line in Fig. 5 shows the expected distribution for a monochromatic atomic beam. This result shows the same symmetry behavior as demonstrated for the unidirectional magneto-optical force [13]. Our results demonstrate the coherent regime of this force.

The atoms emerge from the interaction in the ground state. The magneto-optical beam splitter is nonpolarizing in contrast to, e.g., a Stern-Gerlach beam splitter [7], where the two split parts of the atomic wave function are in orthogonal internal states. The splitting process is coherent as long as at least one period of the phase grating is illuminated coherently. The transverse momentum splitting reaches a principal limit when the Raman-Nath approximation is no longer valid [14]. For the experimental configuration described here this limit corresponds to a symmetric splitting of $100 \hbar k$. However, for $\varphi=45^{\circ}$, the limit is only $25 \hbar k$ in one direction, as the length of the steep gradient of the central eigenvalue $[\lambda / 8$; see Fig. 2 (b)] is reduced by a factor of 2 compared to the symmetric case $\varphi=90^{\circ}$ [see Fig. 2(a)]. The observed discrepancy from the calculated distribution (dashed line in Fig. 5) on the side of larger deflection (see arrow in Fig. 5) is due to the breakdown of the Raman-Nath approximation, which was used for the calculation.

In conclusion, we have presented an experimental demonstration of the diffraction of atoms from a magneto-optical potential. For the expected ratio between the Larmor and Rabi frequencies and orthogonal linear polarizations a symmetric splitting of the atomic wave function was observed. The splitting angle corresponds to a momentum splitting of $42 \hbar k$. The result was compared directly to the diffraction of two-level atoms from a standing wave light field. In contrast to the standing wave diffraction the magneto-optical grating produces an efficient splitting into high order momentum states. For nonorthogonal polarizations $\left(\varphi=45^{\circ}\right)$ a mirror image of the distribution was observed for the sign changes of $B$ or $\varphi$.

In order to demonstrate the coherence of the splitting process, the two parts have to be recombined to observe an interference pattern. The visibility of the interference fringes gives a direct measure of the coherence. In order to observe a reasonable fringe contrast in our case one would have to avoid the optical pumping in front of the magneto-optical interaction region. The need for optical pumping is eliminated by using an atom with a $J=0$ to $J=1$ transition such as $\mathrm{Ca}$ or $\mathrm{Mg}$. In the near field of the magneto-optical grating the triangular phase plate acts as an array of Fresnel biprisms. The interference pattern produced by them would lead to a very narrow spatial feature which could be of interest for lithographic applications.

C.S.A. acknowledges support of the Royal Society's European Science Exchange Programme. This work was supported by the Deutsche Forschungsgemeinschaft.

[1] O. Stern, Naturwissensch. 17, 391 (1929).

[2] O. Carnal and J. Mlynek, Phys. Rev. Lett. 66, 2689 (1991).

[3] D. W. Keith, C. R. Ekstrom, Q. A. Turchette, and D. E. Pritchard, Phys. Rev. Lett. 66, 2692 (1991).

[4] F. Riehle, Th. Kisters, A. Witte, J. Helmcke, and Ch. J. Bordé, Phys. Rev. Lett. 67, 177 (1991).

[5] M. Kasevich and S. Chu, Phys. Rev. Lett. 67, 181 (1991).

[6] Ch. Miniatura, J. Robert, S. Le Boiteux, J. Reinhardt, and J. Baudon, Appl. Phys. B 54, 347 (1992).

[7] T. Sleator, T. Pfau, V. Balykin, O. Carnal, and J. Mlynek, Phys. Rev. Lett. 68, 1996 (1992).

[8] D. S. Weiss, B. C. Young, and S. Chu, Phys. Rev. Lett. 70, 2706 (1993).

[9] See, e.g., P. E. Moskowitz, P. L. Gould, S. R. Atlas, and D. E. Pritchard, Phys. Rev. Lett. 51, 370 (1983); P. L. Gould, G. A. Ruff, and D. E. Prichard, Phys. Rev. Lett. 56, 827 (1986).

[10] T. Pfau, C. S. Adams, and J. Mlynek, Europhys. Lett. 21, 439 (1993).

[11] C. S. Adams, T. Pfau, Ch. Kurtsiefer, and J. Mlynek, Phys. Rev. A 48, 2108 (1993).

[12] O. Carnal, A. Faulstich, and J. Mlynek, Appl. Phys. B 53, 88 (1991); O. Carnal, Ph.D. thesis, ETH Zürich No. 9617, 1992 (unpublished).

[13] R. Grimm, Yu.B. Ovchinnikov, and A. I. Sidorov, Opt. Commun. 84, 18 (1991); R. Grimm, V.-S. Letokhov, Yu.B. Ovchinnikov, and A. I. Sidorow, J. Phys. II (France) 2, 93 (1992).

[14] U. Janicke, C. S. Adams, and M. Wilkens, "Atomic Motion in Optical Potentials" (to be published). 